\title{
OBSTRUCCIÓN PRIMARIA DEL CUELLO VESICAL EN EL VARON.
}

\author{
Pedro Navalón Verdejo 1,2,3, Yoni Pallás Costa', Joaquín Juan Escudero², Felipe Ordoño \\ Domínguez², Francisco Sánchez Ballester², Luis de la Torre Abril2, Elisa Monllor Peidro', \\ Milagros Fabuel Deltoro² y José Antonio Cánovas Ivorra.
}

Unidad de Urodinámica'. Servicio de Urología. Hospital Casa de Salud. Valencia.

Servicio de Urología ${ }^{2}$. Consorcio Hospital General Universitario. Valencia.

Departamento de Cirugía ${ }^{3}$. Facultad de Medicina. Universidad de Valencia. España.

Resumen.- OBJETIVO: Presentar nuestra experiencia en el diagnóstico y tratamiento de esta infrecuente modalidad de obstrucción funcional infravesical.

MÉTODO: Durante los últimos 10 años hemos diagnosticado y tratado a 32 varones afectos de esta entidad. Se evalúan los diversos tratamientos previos recibidos así como el tiempo transcurrido hasta la realización del diagnóstico. Se valoran las manifestaciones clínicas, radiológicas, endoscópicas y urodinámicas así como los resultados obtenidos con la administración de bloqueadores alfa-adrenérgicos y con la incisión transuretral endoscópica del cuello vesical.

RESULTADOS: La edad de los pacientes osciló entre 26 y 48 años (media 38,2). Todos ellos presentaban síntomas significativos del tacto urinario inferior de larga evolución, habiendo recibido diversos tratamientos inespecíficos. El estudio flujométrico evidenció un pico de flujo medio de 9,8 $\pm 3,2 \mathrm{cc} / \mathrm{s}$, siendo la presión del detrusor durante el vaciado de $98 \pm 25 \mathrm{~cm} \mathrm{H} 2 \mathrm{O}$, existiendo un residuo postmiccional significativo en 21 pacientes de $188 \pm 62 \mathrm{cc}$. Con tratamiento médico mediante la administración de bloqueadores alfaadrenérgicos únicamente obtuvimos mejorías subjetivas significativas en 4 pacientes. En 21 casos realizamos incisión unilateral endoscópica del cuello vesical, obteniendo mejorías objetivas y subjetivas en todos ellos.

CONCLUSIONES: La incisión endoscópica del cuello vesical representa el tratamiento de elección de esta disfunción, siendo imperativo informar al paciente del riesgo de eyaculación retrógrada como secuela postoperatoria, ya que esta entidad ocurre en sujetos jóvenes, en los que la fertilidad puede ser una prioridad.

Palabras clave: Obstrucción de cuello vesical. Urodinámica. Desórdenes urinarios.

Summary.- OBJECTIVES: To report our experience on the diagnosis and treatment of this rare type of infravesical functional obstruction.

METHODS: Over the last ten years we diagnosed and treated 32 males with this entity. We evaluate previous treatments and time to diagnosis. Clinical, radiological, endoscopic, and urodynamic features are evaluated, as well as results obtained with the administration of alpha-adrenergic blockers and endoscopic transurethral incision of the bladder neck.

RESULTS: Patient age ranged from 26 to 48 years (Mean 38.2). All patients presented significant long-lasting lower urinary tract symptoms, having received various non-specific treatments. Uroflowmetry showed a peak of mean flow of $9.8+-3.2 \mathrm{cc} / \mathrm{s}$, being the voiding detrusor 
pressure $98+-25 \mathrm{~cm} \mathrm{H} 2 \mathrm{O}$, with significant post void residual volume in 21 patients (188+- $62 \mathrm{cc}$. Medical treatment with alpha-adrenergic blockers only achieved significant subjective improvement in four patients. Twenty one patients underwent unilateral endoscopic bladder neck incision, obtaining objective and subjective improvements all of them.

CONCLUSIONS: Endoscopic bladder neck incision is the treatment of choice of this dysfunction, being imperative to inform the patient of the risk of retrograde ejaculation as a postoperative sequel, since this entity appears in young males, for whom fertility may be a priority.

Keywords: Bladder neck obstruction. Urodynamics. Urinary disorders.

\section{INTRODUCCIÓN}

La obstrucción primaria del cuello vesical (OPCV) constituye una infrecuente modalidad de disfunción de las vías urinarias inferiores que afecta casi exclusivamente a varones jóvenes, siendo su hallazgo en mujeres un hecho excepcional (1). Se trata de una obstrucción funcional de la salida de la vejiga, cuyo diagnóstico lo dificulta la heterogénea sintomatología que provoca, pudiendo variar desde leves síntomas irritativos que simulan una prostatodinia, hasta síntomas severos de obstrucción e incluso de retención, lo que condiciona un frecuente retraso en la instauración de un tratamiento correcto, siendo habitual en estos sujetos una larga historia de consultas a diversos especialistas así como una extensa lista de tratamientos inadecuados, generalmente antibióticos, antiinflamatorios, e incluso ansiolíticos, ya que el cuadro clínico es a menudo confundido con una disfunción miccional psicógena.

Fue Guthrie, en el año 1836 el primero en describir la existencia de una obstrucción orgánica del cuello de la vejiga (2). Posteriormente, en 1913, Young describió un procedimiento que denominó "punch operation" para el tratamiento de la obstrucción del cuello vesical (3). Mas tarde, en 1934, Marion describe un estrechamiento fibroso del cuello de la vejiga y a partir de entonces se utiliza el término de enfermedad de Marion para describir dicha disfunción del cuello y la disfunción del detrusor que le acompaña (4). En 1949, Badenoch refutó el concepto de que el cuello de la vejiga fuese realmente fibrótico y mas estrecho (5), lo que fue posteriormente comprobado en 1986 por Norlen y Blaivas, quienes no hallaron una evidencia histológica de fibrosis ni aumentos en el contenido de colágeno del tejido extraído a hombres con OPCV en el curso de resecciones transuretrales (6).

Se han utilizado diversos términos para identificar este trastorno, tales como enfermedad de Marion, obstrucción primaria del cuello de la vejiga, disinergia del cuello de la vejiga y el detrusor, discinesia del cuello vesical, obstrucción funcional del cuello vesical, disinergia del músculo liso, disinergia del esfínter proximal, disfunción del cuello vesical, obstrucción uretral proximal, etc. Así, la falta de una terminología uniforme otorga crédito a la controversia y a las discrepancias que rodean la comprensión clínica de esta entidad, habiendo aún mas confusión cuando este trastorno se describe con una terminología que evoca una obstrucción estática, no dinámica, por lo que otros términos empleados, tales como hipertrofia del cuello de la vejiga y barra media son inadecuados y no describen exactamente el aspecto funcional de esta entidad.

La detección de la OPCV exige que se la sospeche, por lo que ante un paciente joven con síntomas de moderados a graves de las vías urinarias inferiores debe hacernos tener en consideración la posibilidad de su diagnóstico.

La evidencia objetiva de la obstrucción del tracto de salida en estos pacientes se obtiene con facilidad mediante un estudio urodinámico. Una vez diagnosticada la obstrucción, puede demostrarse la localización de esta a nivel del cuello vesical mediante la realización de estudios videourodinámicos, una cistouretrografía permiccional o bien por medio de una perfilometría uretral miccional. No obstante, el diagnóstico también puede ser realizado con seguridad de forma indirecta a través de los hallazgos urodinámicos de obstrucción del tracto de salida vesical en la situación clínica típica en ausencia de estenosis uretral distal, agrandamiento prostático o disinergia del esfínter estriado (7).

\section{PACIENTES Y MÉTODO}

Revisamos las historias clínicas de 32 pacientes que hemos diagnosticado y tratado en los últimos 10 años de esta infrecuente disfunción miccional. En todos los casos se realizó una anamnesis detallada con especial interés acerca del tiempo de evolución de la sintomatología y de los diversos tratamientos previos recibidos.

El reconocimiento físico puso de manifiesto un normal examen genital y tacto rectal así como una exploración neuro-urológica anodina. 
La exploración urodinámica se practicó con un equipo multicanal MMS UP 2000. En todos los casos se efectuó estudio flujométrico aislado y medida ecográfica del residuo postmiccional así como estudio cistomanométrico durante el llenado vesical y test de presión-flujo durante la fase de vaciado junto a la valoración concomitante electromiográfica perineal con electrodos de superficie.

En todos los casos se realizó estudio ecográfico para evaluar el aparato urinario superior, el volumen prostático y el residuo postmiccional.

Para valorar la localización exacta de la obstrucción, en 25 pacientes se realizó estudio uretrocistográfico retrógrado y permiccional, mientras que en los 7 pacientes restantes se practicó uretrocistoscopia.

Todos los pacientes fueron tratados inicialmente con fármacos bloqueadores alfa-adrenérgicos $15 \mathrm{mg} /$ día de terazosina ó 0,4 mg/día de tamsulosina), reservándose el tratamiento quirúrgico mediante la incisión transuretral del cuello vesical en los pacientes que no respondieron al tratamiento farmacológico.

La intervención quirúrgica fue realizada con un resectoscopio $26 \mathrm{~F}$ armado con asa de Collins. La incisión se efectuó unilateralmente en la posición de las 5 ó 7 horarias del campo endoscópico, practicando un corte profundo desde aproximadamente un centímetro distal al meato ureteral sobre el cuello vesical hasta llegar a la proximidad del verumontanum, profundizando hasta visualizar la grasa pericervical y las fibras periprostáticas. Durante el postoperatorio se insertó una sonda de Foley que se retiró cuando cedió la hematuria, generalmente en las primeras 24 a 48 horas. Solamente en un paciente con hematuria prolongada, se mantuvo la sonda durante cinco días.

La primera revisión tras el tratamiento se realizó a los tres meses, evaluando el número de pacientes que refirieron mejorías subjetivas, valorando asimismo las variaciones en la flujometría y en el residuo postmiccional, comparando estadísticamente los resultados cuantitativos mediante la prueba $t$ de Student y los cualitativos mediante el test del chi-cuadrado, definiendo la significación estadística cuando $p<0$ 05.

\section{RESULTADOS}

Todos los pacientes fueron varones, con edades comprendidas entre 26 y 48 años (media 38'2) y en la totalidad de ellos había una larga historia de consultas médicas a diversos especialistas, con un tiempo medio aproximado de evolución de $4^{\prime} 1$ años (rango: 2 - 7), refiriendo haber sido tratados con tandas de antibiótico 30 de ellos (94\%), fitoterapia 29 (91\%), antiinflamatorios no esteroideos $16(50 \%)$, y benzodiazepinas 12 (37\%).

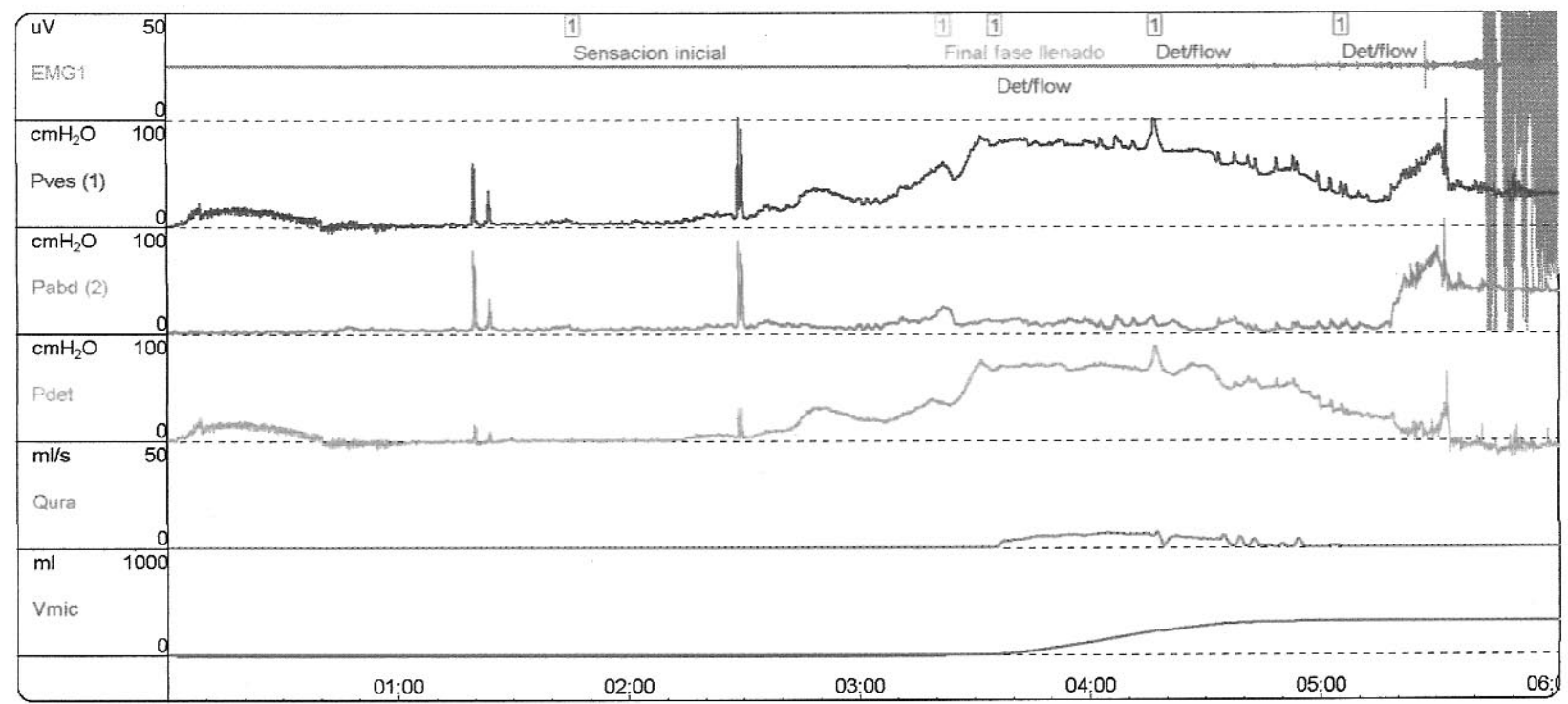

FIGURA 1. Trazado urodinámico característico: bajo flujo con alta presión y normalidad electromiográfica perineal. 
La flujometría libre evidenció un índice de flujo bajo, con un pico de flujo de $9,8 \pm 3,2 \mathrm{cc} / \mathrm{s}$, existiendo un residuo postmiccional significativo en 21 pacientes de $188 \pm 62 \mathrm{cc}$ de media. Durante la fase cistomanométrica de llenado se observó la presencia de inestabildad vesical en $9(28 \%)$ de los pacientes. El test de presión-flujo evidenció bajo flujo y alta presión en todos los casos, siendo la presión del detrusor durante el vaciado de $98 \pm 25 \mathrm{~cm} \mathrm{H} 20$. El estudio electromiográfico puso de manifiesto en todos los casos una correcta relajación perineal durante la fase de vaciado (Figuras 1 y 2 ).

La ecografía, además de evidenciar un residuo postmiccional significativo en 21 pacientes, puso de manifiesto un volumen prostático normal así como la normalidad del aparato urinario superior en todos los casos.

En 25 pacientes se practicó estudio radiológico vesicouretral, evidenciando en todos ellos un normal calibre de la uretral distal, así como la insuficiente apertura del cuello vesical durante la cistouretrografía permicional (Figura 3). En los 7 pacientes restantes, se practicó uretrocistoscopia para descartar la estenosis uretral como causa de la obstrucción.

En la primera revisión realizada aproximadamente a los tres meses de la instauración del tratamiento, únicamente 4 pacientes $(12 \%)$ refirieron mejoría sintomática subjetiva con la administración de bloqueadores alfa-adrenérgicos (Tabla I) y otros 6 enfermos abandonaron la medicación al no haber observado un alivio adecuado de los síntomas. Asimismo, tampoco hubo diferencias estadísticamente significativas en los picos de flujo $\left(10^{\prime} 1 \pm 3^{\prime} 3 \mathrm{cc} / \mathrm{s}\right)$,

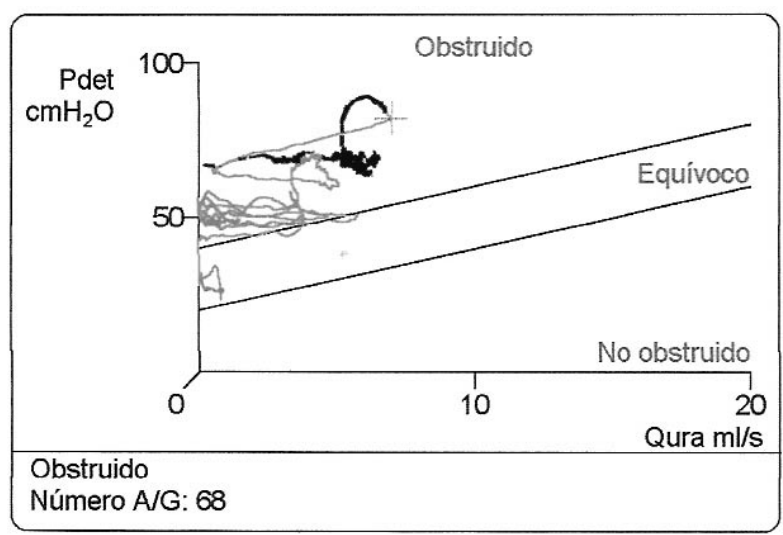

FIGURA 2. Gráfico detrusor/flujo según ICS: trazado típico de obstrucción con bajo flujo y alta presión del detrusor: ni en el volumen del residuo postmiccional (195 \pm $52 \mathrm{cc}$ ). No obstante, en los 21 casos que realizamos incisión endoscópica del cuello vesical, en todos ellos hubo una alivio subjetivo de los síntomas, así como una mejoría significativa del pico de flujo $(22,4 \pm$ $6,5 \mathrm{cc} / \mathrm{s})$ y del residuo postmiccional $(55 \pm 43 \mathrm{cc})$. Ninguno de los pacientes presentó trastornos de la erección tras la intervención, y en tres de ellos apareció eyaculación retrógrada como secuela, refiriendo otros tres pacientes importante disminución en el volumen del eyaculado.

\section{DISCUSIÓN}

Esta disfunción se encuentra casi exclusivamente en hombres jóvenes y de mediana edad, siendo típico que los pacientes manifiesten síntomas miccionales tanto obstructivos como irritativos de larga evolución, habiendo recibido múltiples tratamientos, y frecuentemente se les ha realizado un diagnóstico erróneo de disfunción miccional psicógena debido al hallazgo de una próstata normal en el examen rectal, un volumen de orina residual habitualmente despreciable y un aspecto endoscópico normal de la vejiga.



FIGURA 3. Cistouretrografía permiccional: Insuficiente apertura del cuello vesical con uretra distal normal. 
La etiología de esta entidad no se conoce con exactitud; sin embargo, diversas publicaciones apuntan hacia la existencia de una anomalía en el desarrollo de la musculatura del cuello vesical y del detrusor. Así, Turner-Warwick observó que el verdadero cuello vesical no es un músculo concéntrico aparte, sino que mas bien está compuesto por una disposición de las fibras de los músculos lisos que se extienden desde el trígono distalmente hasta el nivel del verumontanum, lo que representa un mecanismo oclusivo y competente constituyendo un "esfínter interno" del detrusor en reposo. El comportamiento del cuello vesical depende de la función estable del detrusor, puesto que una contracción del detrusor hará abrirse el cuello infundibulizándolo a modo de embudo (8). Tanagho afirmó que la apertura del cuello vesical precede inmediatamente al establecimiento de una contracción del detrusor y que ésta es provocada por un reflejo (9). Turner-Warwick describió por primera vez la obstrucción del cuello de la vejiga como una insuficiencia disinérgica congénita del cuello vesical para abrirse, afirmado que esto se debe a una disposición disfuncional de la musculatura del cuello que tiene su origen durante el desarrollo y resulta en una abertura inadecuada provocada por un reflejo anormal. (8).

Bates y colaboradores (10) evaluaron la dinámica de vaciado miccional en pacientes afectos OPCV y la compararon con sujetos normales, no hallando diferencias entre ambos grupos en las presiones intrínsecas del cuello durante el reposo. No obstante, sí que advirtieron una diferencia significativa en las presiones vesicales máximas que se generan durante la micción, concluyendo que la obstrucción debe ser de naturaleza más funcional que estructural puesto que las presiones en reposo eran similares en los dos grupos. Asimismo, el hecho de que los perfiles de presión obtenidos durante la micción fueran más altos en los sujetos afectos de OPCV implica que el cuello vesical se contrae durante el vaciado, lo que da a entender que la OPCV es un fenómeno activo.

Posteriormente, otros estudios (11) confirmaron el predominio de receptores alfa adrenérgicos dentro del trígono y uretra posterior, demostrando que la inervación simpática desempeña un papel fundamental en el control de la presión de la uretra proximal. En estos estudios, la utilización de fentolamina para el bloqueo de los receptores alfa adrenérgicos dieron como resultado una disminución importante en los perfiles de presión uretral. Esta respuesta farmacológica indujo la teoría de la función simpática exagerada como posible factor etiológico de la obstrucción de la salida de la vejiga, así como las implicaciones médicas en el tratamiento de dicha obstrucción.

Por otra parte, nuevas investigaciones (12) estudiaron la posibilidad de una causa de origen neurológico de la OPCV y examinaron la inervación del cuello vesical en pacientes con este trastorno, hallando una mayor densidad de inervación simpática en el cuello vesical de estos pacientes. No obstante, se debe tener en cuenta la posibilidad de que estos hallazgos puedan reflejar las consecuencias de otra patología oculta y no del problema principal. (13).

Mientras el mecanismo etiológico primario causante de la OPCV sigue sin conocerse, TurnerWarwick realizó las siguientes puntualizaciones al respecto: 1) el cuello de la vejiga se distingue morfológicamente de las fibras del detrusor proximal en que éstas consisten en pequeñas agrupaciones de haces musculares y su inervación es principalmente adrenérgica; 2) como ha demostrado Tanagho, la abertura del cuello vesical se realiza por medio de un reflejo y antecede al aumento de la presión asociada a la contracción detrusoriana; 3) la abertura "disinérgica" del cuello de la vejiga es una disfunción y no

TABLA I. EVALUACIÓN DE LOS RESULTADOS.

\begin{tabular}{|lccc|}
\hline & Preoperatorio & alfa-bloq & Incisión TU \\
\hline Pico de flujo $(\mathrm{cc} / \mathrm{s})$ & $9,8 \pm 3,2$ & $10,1 \pm 3,3$ & $22,4 \pm 6,5^{*}$ \\
Residuo postmiccional $(\mathrm{cc})$ & $188 \pm 62$ & $195 \pm 52$ & $55 \pm 43^{*}$ \\
Presión máx. detrusor $\left(\mathrm{cm} \mathrm{H}_{2} \mathrm{O}\right)$ & $98 \pm 25$ & - & - \\
Mejorías subjetivas $\left(\mathrm{n}^{\circ}\right.$ pacientes) & - & 4 & 21 * \\
Eyaculación retrógrada ( ${ }^{\circ}$ pacientes) & - & - & 3 \\
\hline
\end{tabular}

(*) significación estadística 
se debe considerara neuropática o resultado de una neuropatía manifiesta; 4) la abertura del cuello de la vejiga se asocia a la contracción del detrusor y no existe un umbral o una amplitud de presión a la que se deba llegar, y 5) la mecánica de la disfunción del cuello de la vejiga rara vez cambia, ya que la mayor parte de su comportamiento característico puede indagarse hasta la infancia si realizamos una anamnesis meticulosa. No obstante, como ocurre con muchas anomalías congénitas que no se manifiestan clínicamente hasta la edad adulta, también en la entidad que nos ocupa, la gravedad de los síntomas puede empeorar con la edad, quizás como consecuencia de la descompensación del detrusor secundariamente a la obstrucción crónica o un crecimiento prostático coexistente. Asimismo, es muy infrecuente que un cuello vesical sinérgico se convierta en disfuncional, a excepción de las neuropatías adquiridas como, por ejemplo, la vejiga de los diabéticos (8).

La forma de presentación clínica de la enfermedad es muy variada dependiendo del grado de obstrucción, siendo pocos los pacientes que refieren síntomas clásicos de obstrucción de la salida vesical, ya que las pautas obstructivas de vaciado que presentan estos sujetos les han acompañado durante toda la vida y por tanto las consideran normales. Las manifestaciones clínicas en estos pacientes pueden variar desde la incapacidad de orinar en público hasta el hallazgo de un elevado residuo postmiccional que puede llevar a un estado de retención cuando la vejiga se distiende demasiado. Igualmente, muchos pacientes refieren síntomas irritativos como consecuencia de la inestabilidad del detrusor secundaria a la obstrucción crónica.

Es también habitual en estos pacientes la existencia de un historial de infecciones recurrentes de las vías urinarias (normalmente no documentadas), habiendo sido a menudo diagnosticados erróneamente de prostatitis crónica, prostatodinia y anomalías de la relajación del suelo pélvico, siendo sometidos frecuentemente a largos tratamientos con antibióticos, agentes antiinflamatorios, ansiolíticos y relajantes musculares e incluso modificaciones de sus dietas, como ocurrió en la práctica totalidad de nuestros pacientes, que tuvieron un diagnóstico erróneo de alguna forma de enfermedad prostática inflamatoria, por lo que somos de la opinión junto con otros autores (13) que ante cualquier varón con un historial de infección de las vías urinarias debe sospecharse la existencia de una obstrucción, y siempre se debe considerar la posibilidad de una OPCV en los menores de 50 años.

También es frecuente en estos pacientes el diagnóstico erróneo de obstrucción secundaria a cre- cimiento prostático, aunque también es posible que coexistan ambas patologías en el mismo sujeto. Así, cuando se desarrolla un agrandamiento de la glándula prostática en los individuos con este problema, se produce una obstrucción doble que Turner-Warwick identificó con el término de "próstata atrapada" para describir la combinación de engrosamiento prostático en coexistencia con la OPCV, en donde los lóbulos de la próstata no pueden expandirse en el cuello de la vejiga y por lo tanto lo hacen en la uretra, lo que condiciona que un aumento relativamente pequeño del volumen de la próstata provoque graves síntomas de vaciado (8).

El examen físico no es de gran ayuda para la identificación de esta entidad, pues la exploración neuro-urológica suele ser normal (14). La próstata presenta generalmente un escaso relieve durante la exploración rectal; sin embargo, el tamaño de la próstata no está relacionado con el flujo ni con el grado de obstrucción. También la uretrocistoscopia ofrece unos beneficios limitados ya que brinda una imagen estática de un proceso dinámico, no pudiéndose por tanto evaluar correctamente por observación endoscópica el comportamiento del mecanismo de apertura del cuello vesical (8). No obstante, el examen endoscópico puede proporcionar pistas indirectas tales como el grado de hipertrofia del detrusor y de trabeculación (relacionada habitualmente con la inestabilidad mas que con la obstrucción), así como la posibilidad de cierto engrosamiento prostático simultaneo, pudiendo también descartar la posible existencia de una estenosis uretral distal $(13,15)$.

Las técnicas de diagnóstico por imagen tienen un valor relativo en el diagnóstico de esta entidad. La ecografía, además de permitir determinar el volumen prostático, valora el residuo postmiccional así como el estado del aparato urinario superior. Asimismo, el estudio radiológico vesicouretral permite descartar la estenosis uretral distal como causa de la obstrucción así como la falta de apertura del cuello vesical durante la cistouretrografia miccional. (Figura 3).

Unicamente el estudio urodinámico será capaz de asignar el carácter obstructivo a los datos clínicos y endoscópicos, constituyendo la flujometría, combinada con la valoración de residuo postmiccional, una exploración fundamental, ya que permite obtener un registro gráfico de la pauta habitual de vaciado miccional del paciente, pues el relato de dicha pauta por el propio sujeto durante la anamnesis suele inducir a error, ya que habitualmente está acostumbrado a este trastorno desde la niñez, por lo que suele considerarlo normal. Asimismo, la flujometría es de gran utilidad para evaluar los resultados del tratamiento. 
Los índices de flujo urinario máximo (Qmax) generados por los pacientes afectos de OPCV oscilan entre 8,9 y $11,8 \mathrm{ml} / \mathrm{s}$ según diversos autores revisados $(13,15,16)$, siendo en nuestra experiencia de $9,2 \pm 3,1$. No obstante, el hallazgo de un bajo flujo y un residuo postmiccional elevado no siempre son el resultado de una obstrucción del tracto de salida vesical, siendo necesario la determinación simultanea de la presión vesical de vaciado y de los índices de flujo para dar un diagnostico definitivo.

También, es importante tener en cuenta que el registro aislado del flujo urinario en pacientes jóvenes puede ser ambiguo, pues algunos pacientes afectos de OPCV son capaces de generar índices de flujo normales mediante elevadas presiones del detrusor, y si no se evalúa ésta última, la OPCV puede pasar sin ser diagnosticada $(8,13)$; en consecuencia, la utilización de equipos de urodinamia multicanal con registro simultaneo de las presiones intravesicales e intrabdominales durante las fases de llenado y vaciado, sigue siendo la forma idónea para el diagnóstico de obstrucción, constituyendo el test de presión-flujo una prueba imprescindible para la evaluación urodinámica de obstrucción de la salida vesical. Así, una elevada presión del detrusor junto con una disminución de los índices de flujo y la relajación del esfínter externo de la uretra en el electromiograma confirman dicha obstrucción.

Asimismo, en los sujetos afectos de OPCV es frecuente el hallazgo de contracciones involuntarias del detrusor durante la fase cistomanométrica de llenado, siendo esta inestabilidad la responsable de los síntomas irritativos que experimentan estos pacientes, lo que ocurrió en $9(28 \%)$ de nuestros casos.

La videourodinámica, con el complemento de la fluoroscopia simultanea proporciona la capacidad de determinar con exactitud la localización de la obstrucción durante el vaciado vesical; y aunque algunas publicaciones han sugerido el empleo de la perfilometría de la presión dinámica vesicouretral durante la micción para una mejor localización del nivel de obstrucción (17), dicha técnica, aunque proporciona muchos detalles, suele requerir una gran experiencia, precisando de gran minuciosidad para su realización, necesitando también de la fluoroscopia, por lo que sus beneficios con respecto a una evaluación videourodinámica no parecen estar muy claros (13). Los estudios videourodinámicos también proporcionan imágenes fluoroscópicas del esfínter externo, constituyendo la combinación de la electromiografía y la visualización fluoroscópica vesicouretral durante el vaciado una prueba de incuestionable valor en situaciones complejas (13). No obstante, como es nuestro caso, existen muchos grupos de trabajo que no poseen equipo de videourodinámica, lo cual no es óbice para poder establecer el diagnóstico de OPCV con seguridad, ya que éste se puede realizar de forma indirecta a través de los hallazgos urodinámicos de obstrucción del tracto de salida vesical en la situación clínica típica en ausencia de estenosis uretral distal, agrandamiento prostático o disinergia del esfínter estriado. $(7,18)$.

En lo que se refiere al tratamiento de esta disfunción, la elevada densidad de receptores alfaadrenérgicos existentes en el cuello vesical hace razonable enfocar su tratamiento farmacológicamente intentando controlar el sistema simpático sobreactivo mediante el empleo de agentes alfa-bloqueadores (19). Desafortunadamente, como hemos podido constatar en nuestra experiencia, donde el 19\% de los pacientes abandonaron el tratamiento por no haber observado un alivio adecuado de los síntomas, los resultados terapéuticos con el empleo de dichas sustancias han sido desalentadores, y la mayor parte de los estudios revisados propugnan la intervención quirúrgica como la mejor opción terapéutica (1, 20-22), siendo la incisión transuretral del cuello de la vejiga el tratamiento de elección propugnado por la mayoría de los autores consultados, ya que la resección del cuello, además de tener una mayor morbilidad, presenta un mayor riesgo de esclerosis postoperatoria (23).

Nosotros preferimos la realización de una sola incisión en la posición de las 5 o de las 7 del campo endoscópico, practicando un corte profundo desde un punto proximal al cuello vesical sobre el trígono hasta llegar a la proximidad del verumontanum. La incisión unilateral simplifica el procedimiento de tal modo que puede ser realizado bajo anestesia local-sedación y en régimen ambulatorio (24); asimismo, además de abreviar el tiempo quirúrgico disminuye la probabilidad de hemorragia postoperatoria y también minimiza la potencial presentación de eyaculación retrógrada (23), de cuyo riesgo se debe de informar al paciente antes de la intervención, ya que se trata de pacientes jóvenes donde la pérdida de esta función puede tener trascendencia.

\section{CONCLUSIONES}

Los pacientes afectos de OPCV son a menudo erróneamente diagnosticados al ser confundida su sintomatología con algún tipo de patología inflamatoria prostática e incluso con una disfunción miccional psicógena, por lo que su detección exige un elevado índice de sospecha. Por lo tanto, ante cualquier varón con una larga historia de infecciones de las vías 
urinarias debe valorarse la existencia de obstrucción, y siempre se debe considerar la posibilidad de una OPCV en los menores de 50 años.

La incisión endoscópica del cuello vesical representa el tratamiento de elección de esta disfunción, siendo imperativo informar al paciente del riesgo de eyaculación retrógrada como secuela postoperatoria, ya que esta entidad ocurre en sujetos jóvenes, en los que la fertilidad puede ser una prioridad.

\section{BIBLIOGRAFÍA y LECTURAS RECOMENDADAS ( ${ }^{*}$ lectura de interés $y^{* *}$ lectura fundamental)}

1. BLAIVAS, J.G.; FLISSER, A.J.; TASH, J.A.: "Treatment of primary bladder neck obstruction in women with transurethral resection of the bladder neck”. J. Urol., 171: 1172, 2004.

2. GUTHRIE, G.D.: "On the anatomy of diseases of the urinary and sexual organs". Ed Churchill, chapter 4, p 60. London, 1836.

3. YOUNG, H.H.: "A new procedure (punch operation) for small prostatic bars and contracture of the prostatic orifice". JAMA, 60: 253, 1913.

4. MARION, G.: "Maladie du col vesical". J. Urol., 37: 5, 1934.

5. BADENOCH, A.W.: "Congenital obstruction at the bladder neck". Annals of the Royal College of Surgeons, 4: 295, 1949.

6. NORLEN, L.J.; BLAIVAS, J.G.: "Unsuspected proximal urethral obstruction in young and middle aged men". J. Urol., 135: 972, 1986.

*7. WEIN, A.J.: "Disfunción neuromuscular de las vias urinarias inferiores y su manejo". Walsh PC.

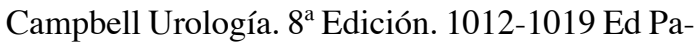
namericana; Madrid. 2004.

**8. TURNER-WARWICK, R.T.; WHITESIDE, C.C.; WORTH, P.H. y cols.: "A urodynamic view of the clinical problems associated whit bladder neck dysfunction and its treatment by endoscopic incision and transtrigonal posterior prostatectomy". Br. J. Urol., 45: 44, 1984.

9. TANAGHO, E.A.: "The anatomy and physiology of micturition". Clin. Obst. and Gyn., 5: 3, 1973.

10. BATES, C.P.; ARNOLD, E.P.; GRIFFTHS, D.J.: "The nature of the abnormality in bladder neck obstruction”. Br. J. Urol., 47: 651, 1973.

11. AWAD, S.A.; DOWNIE, J.W.; LYWOOD, D.W. y cols.: "Sympathetic activity in the proximal urethra in patients with urinary obstruction". J. Urol., 115: 545, 1976.
12. CROWE, R.; NOBLE, J.; ROBSON, T. y cols.: "An increase of neuropeptide Y but not nitric oxide synthase-inmunoreactive nerves in the bladder neck from male patients with bladder neck dyssynergia”. J. Urol., 154: 1231, 1995.

**13. KRAUS, S.R.; SMITH, C.P.; BOONE, T.B.: "La obstrucción primaria del cuello de la vejiga en el hombre". Ball TP (Ed): AUA Update Series; 4955; Ed Medical Trends SL; Barcelona; 2001.

**14. SALINAS, J.: "Obstrucción funcional del tracto urinario inferior". Salinas J, Romero J (Ed): Urodinámica Clínica 2a Ed; 229-246; Jarpyo Editores; Madrid; 1995.

15. KAPLAN, S.A.; IKEUCHI, E.F.; SANTAROSA, R.P. y cols.: "Etiology of voiding dysfunction in men less than 50 years of age". J. Urol., 47: 836, 1996.

16. SURI, A.; SRIVASTAVA, A.; SINGH, K.J. y cols.: "Endoscopic incision for functional bladder neck obstruction in men: long-term outcome". J. Urol., 66: 323, 2005.

17. YALLA, S.V.; WATERS, W.B.; SNYDER, H. y cols.: "Urodynamics localization of isolated bladder neck obstruction in men. Studies with micturitional vesicourethral static pressure profile". J. Urol., 125: 677, 1981.

18. PENA, J.M.; LEON, E.; CONGREGADO, y cols.: "La urodinámica en la estenosis de uretra". Salinas J, Romero J (Ed): Urodinámica Clínica: Disfunciones vesicouretrales. 3a Ed; 355-372; Luzan 5 SA Editores; Madrid; 2002.

*19. YANG, S.S.; WANG, C.C.; HSIEH, C.H. y cols.: "Alpha-1-adrenergic blockers in young men with primary bladder neck obstruction”. J. Urol., 168: $571,2002$.

20. TROCKMAN, B.A.; GERSPACH, J.; DMOCHOWSKI, R. y cols.: "Primary bladder neck obstruction: Urodynamic findings and treatment results in 36 men”. J. Urol., 156: 1418, 1996.

21. KAPLAN, S.A.; TE, A.E.; JACOB, B.Z.: "Urodynamic evidence of vesical neck obstruction in men with misdiagnosed chronic nonbacterial prostatitis and the therapeutic role of endoscopic incision of the bladder neck". J. Urol., 152: 2063, 1994.

22. CHRISTENSEN, M.G.; NORDLING, J.; ANDERSEN, J.T. y cols.: "Functional bladder neck obstruction. Results of endoscopic bladder neck incision in 131 consecutive patients". Br. J. Urol., 57: 60, 1985.

23. BLASCO, J.E.; GONZALVO, V.; NAVALON, P. y cols.: "Trigonocervicoprostatotomía endoscópica: Nuestra experiencia". Actas Urol. Esp., 17: 315, 1993.

24. NAVALON, P.; ZARAGOZA, C.; ORDOÑO, F. y cols.: "Tratamiento endoscópico de la obstrucción del cuello vesical del varón en CMA". Cir. May. Amb., 10: 126, 2005 